Cristina Tutuianu ORCID iD: 0000-0003-0754-9584

\title{
Is adenosine useful for the identification of atrial fibrillation triggers?
}

Cristina Tutuianu MD, Robert Pap MD, PhD, Tamas Riesz MD, Gabor Bencsik MD, $\mathrm{PhD}$, Attila Makai MD, PhD and Saghy Laszlo MD, PhD

University of Szeged, Hungary

Short title: Adenosine for atrial fibrillation induction.

Corresponding Autor:

Cristina Tutuianu, MD,

$2^{\text {nd }}$ Department of Medicine and Cardiology Center

University of Szeged, Hungary

6725 Szeged, Semmelweis str. 6

Phone: +3662545220

Fax: +3662544568

Email: tutucris2008@gmail.com

Abstract:

Introduction: Both isoproterenol (Iso) and adenosine (Ado) are used to induce atrial fibrillation (AF) in the electrophysiology lab. However, the utility of Ado has not been systematically established.

Objective: The purpose of this study was to compare Ado to Iso for the induction of paroxysmal AF.

Methods: Forty patients (16 women, mean age: $60 \pm 12$ years) with paroxysmal AF, presenting for ablation were prospectively included of whom 36 (90\%) received Ado (18-36 mg) and/or Iso (3-20 $\mu \mathrm{g} / \mathrm{min}$ incremental dose) in a randomized order (26 (72\%) received both drugs).

Results: AF was induced with Iso in 15/32 (47\%) and with Ado in 12/30 (40\%) patients $(\mathrm{p}=0.9)$. Iso-triggered AF started from the left pulmonary veins (PVs) in $11 / 15$ (73\%), from the right PVs in 3/15 (20\%), from the coronary sinus (CS) in 1/15

This article has been accepted for publication and undergone full peer review but has not been through the copyediting, typesetting, pagination and proofreading process, which may lead to differences between this version and the Version of Record. Please cite this article as doi: $10.1111 /$ jce.13779.

This article is protected by copyright. All rights reserved. 
(7\%) cases. Ado-induced AF episodes originated from the left PVs in 6/12 (50\%), from the right atrium (RA) in 4/12 (33\%), from the CS in 2/12 (17\%) cases. Altogether Iso-induced AF was more likely initiated from the PVs (93\%), compared to Ado $(50 \%)(\mathrm{p}=0.02)$. Ado-induced non-PV triggers were not predictive of arrhythmia recurrence after PV isolation.

Conclusion: Ado much more frequently induces non-PV triggers, especially from the RA. The clinical significance of these foci, however, is questionable.

Keywords: adenosine, isoproterenol, triggers, atrial fibrillation, pulmonary vein.

Disclosures: None

Drs. Tutuianu and Pap had equal contribution to the manuscript.

\section{Introduction}

Elimination of initiating triggers has become the cornerstone of atrial fibrillation (AF) treatment. Most of these triggers reside in the pulmonary veins (PV) (1); however, non-PV triggers are an important source of recurrence after PV isolation. Triggers can be identified during the electrophysiology study if spontaneously occurring or can be induced by drug challenge. Due to the spurious nature of spontaneous triggers and the laboriousness of AF provocation, empirical isolation of all PVs has become the standard in AF ablation, despite the fact that selective isolation of only the triggering PV can achieve similar success in selected patients (2). Even if total PV isolation is pursued as a first step, identification of non-PV triggers gains importance when $\mathrm{AF}$ occurs despite isolated PVs $(3,4)$. The role of high dose isoproterenol (Iso) infusion to elicit AF triggers is well established $(5,6)$. Besides Iso, adenosine (Ado) or adenosinetriphosphate (ATP) is increasingly used for the induction of AF, despite the lack of systematic studies on the sensitivity and specificity of these drugs. We sought to determine the utility of Ado in identifying triggers of paroxysmal AF by comparing it to Iso in a prospective, randomized study.

This article is protected by copyright. All rights reserved. 


\section{Methods}

\section{Study Population}

Sample size estimation was based on a previous pilot analysis of retrospective data (7). In this, we found marked differences in the location of triggers disclosed by Iso and Ado: only PV triggers were induced with Iso, while non-PV triggers were seen in 88\% of those receiving Ado also. Expecting less pronounced a difference: 20\% versus $80 \%$ non-PV trigger rate with Iso versus Ado, respectively, and an $80 \%$ induction rate for both drugs, the sample size for an alpha value of 0.05 and a power of 0.8 would be 15 patients tested with each drug. Due to dropouts and a lower induction rate during the study, altogether 40 patients were enrolled, with the aim being to test at least 30 patients with each drug.

All 40 patients (16 women, mean age $60 \pm 12$ years) had paroxysmal AF, and were referred for catheter ablation. The study was approved by the Institutional Review Board of the University of Szeged (no. 41-83). All patients gave their written informed consent to participate in the study. Exclusion criteria were previous left atrial (LA) catheter ablation or open heart surgery, persistent AF, severe valvular heart disease or LA thrombus.

Patients were prospectively included and received Ado and Iso for induction in a randomized order.

\section{Electrophysiologic Procedure}

Patients were on oral anticoagulation for at least 3 weeks before the ablation and all antiarrhythmic drugs were discontinued for at least five half-lives except amiodarone, 
which was discontinued 1 month prior to the procedure. Patients presented in the lab in sinus rhythm or in AF for ablation. In case of AF prior to the induction protocol, direct current cardioversion (DCCV) was performed. The study was performed under light conscious sedation with midazolam \pm fentanyl, as described previously $(8,9)$. Briefly, after femoral venous access two decapolar steerable catheters (interelectrode spacing 2-5-2 mm, Dynamic Deca, Bard Electrophysiology, Lowell, MA, USA) were positioned in the coronary sinus (CS) and the posterolateral right atrium with the distal pole in the superior vena cava. Double transseptal punctures were performed to access the left atrium (LA). Intracardiac echocardiography (ICE) (AcuNav, Acuson Corp., Mountain View, CA, USA) was used to guide the transseptal puncture and catheter positioning. A decapolar circular mapping catheter (CMC) (Inquiry Optima, St Jude Medical, Irvine, CA, USA) was positioned at the left PV antrum overlying both left PVs, and a $3.5 \mathrm{~mm}$ irrigated-tip mapping catheter (Navistar Thermocool, Biosense Webster, Diamond Bar, LA, USA) was positioned on the right PV carina. (Figure 1).

\section{Drug Challenge}

If the patient presented in AF, DCCV was performed to restore sinus rhythm and evaluate for spontaneous re-initiation and identifying post cardioversion AF triggers. If the presenting rhythm was sinus or AF was not spontaneously reinitiated after the cardioversion, we proceeded with the drug challenge.

Isoproterenol (Iso) was infused via a short femoral venous sheath in incremental doses starting at $3 \mu \mathrm{g} / \mathrm{min}$ and increasing after $3-5$ minutes to $5 \mu \mathrm{g} / \mathrm{min}, 10 \mu \mathrm{g} / \mathrm{min}$, 15ug/min, and a maximum dose of $20 \mu \mathrm{g} / \mathrm{min}$, until induction of AF or intolerable side effects occurred.

This article is protected by copyright. All rights reserved. 
Adenosine (Ado) was administered into the right atrium (RA) via one long transseptal sheath that was pulled back in the RA. A quick bolus of $18 \mathrm{mg}$ was given flushed with 5-10 $\mathrm{ml}$ of saline. A second dose of $36 \mathrm{mg}$ was administered if AF was not induced after the first dose. Ectopic activity triggering an AF episode was identified and the origin determined based on the endocardial activation sequence, as previously described $(8,9)$. Triggers of AF were considered to arise from the left PVs if the earliest activation and LA-PV electrogram reversal was recorded on the CMC and from the right PVs if the earliest atrial activation was recorded on the mapping catheter positioned at the right PV carina. If earliest atrial activation during bursts of ectopic activity initiating AF was recorded at any of the CS or RA bipoles, then these structures were identified as the origin of triggers. (Figure 2)

The order in which the two drugs were administered (Iso first or Ado first) was randomized in a 1:1 fashion. When sustained AF was induced and did not terminate after a few minutes, DCCV was performed and the second drug was administered after a 5-minute waiting period. In cases when immediate recurrence of AF (IRAF) occurred after DCCV, drug challenge was terminated and we proceeded with ablation. We determined the effectiveness of the two drugs in inducing AF and compared them with each other and spontaneous AF episodes in terms of the location of AF triggers.

\section{Catheter ablation}

After creation of a 3-dimensional electroanatomic shell of the LA, circumferential, irrigated radiofrequency ablation lesions were created around ipsilateral PVs using the mapping catheter, with the endpoint of electrical isolation manifested in exit and entrance block between LA and PVs. The decision to selectively isolate only the left or right PVs - whichever were shown to be arrhythmogenic during drug challenge - or 
empirically isolate all 4 PVs was at the discretion of the operator. However, in case of a redo procedure all 4 PVs were isolated, irrespective of the initial approach.

\section{Statistical Analysis.}

Continuous variables are expressed as mean \pm SD and compared using Student's t test. The categorical variables are reported as percentage and compared using Chi-square analysis. All statistical analyses were performed using the SPSS software version 16 (IBM Inc., NY, USA). A p<0.05 was considered statistically significant.

\section{Results}

Four (10\%) patients could not receive any drug challenge because their spontaneous AF ongoing at the commencement of the procedure restarted immediately after DCCV. Eighteen of the remaining 36 patients were randomized to receive Iso first and 18 Ado first. In case of 10 (28\%) patients the second drug was not given, because the AF induced by the first drug spontaneously restarted after DCCV. Therefore 36 (90\%) patients received their first and 26/36 (72\%) patients the second drug. Altogether 32 patients received Iso and 30 patients Ado (30 received 18 mg, 21 received 18 and 36 mg). AF was induced with Iso in 15/32 (47\%) and with Ado in 12/30 (40\%) patients $(\mathrm{p}=0.9)$. Iso-triggered AF started from the left PVs in 11 (73\%), from the right PVs in 3 (20\%), from the CS in 1 (7\%) cases (Figure 3A). Ado-induced AF episodes originated from the left PVs in 6 (50\%), from the RA in 4 (33\%), from the CS in 2 (17\%) cases (Figure 3B). Altogether Iso-induced AF was more likely initiated from the PVs (93\%), compared to Ado (50\%) ( $\mathrm{p}=0.02)$.

Of the 26 (72\%) patients (Figure 4., Table 1.) who received both drugs, AF could not be induced by drug challenge in 13 (50\%). Iso triggered AF in 9/26 (35\%) patients; all 
triggers were localized to the PVs (7 left, 2 right PVs). Ado was effective at inducing AF in 8/26 (31\%), 2 from left PVs and 6 from non-PV sites (4 RA, 2 CS, $p<0.01$ versus Iso). Both drugs induced AF in 4/26 (15\%) of these cases. In 2 of those 4 patients triggers originated from the left PVs with both Iso and Ado, while in the remaining two cases there was discordance between the two drugs, Ado manifesting non-PV triggers.

Fourteen (35\%) patients had spontaneous AF during the procedure, 13 (93\%) originated from the PVs (9 left, 4 right PVs), and only one from the CS. Ten of these cases received one or both drugs. Iso reproduced left or right PV triggers in 6/7 cases and was ineffective in one. Ado reproduced left PV triggers in 4/7 cases, but was ineffective in 3 . This results in a sensitivity to reproduce spontaneous triggers of $86 \%$ for Iso and 57\% for Ado.

After drug testing 38 of 40 (95\%) patients underwent PVI. Two patients - for whom initially a selective PVI was planned - did not have an ablation (one was noninducible and one had RA trigger on Ado). Thirty of the remaining 38 (79\%) received empirical isolation of all 4 PVs, while 8 (21\%) patients a selective PVI of arrhythmogenic PVs. Ten of 38 (26\%) underwent a second PVI procedure with the aim of 4-PV isolation, because of recurrence of AF. After the last procedure, 32 of 38 (84\%) ablated patients were free of recurrence during $16 \pm 9$ months of follow-up.

Of the 14 patients who had PV triggers disclosed by Iso infusion, one was lost to follow-up, and the rest had no AF recurrence after the last PVI. In case of the only patient with a non-PV trigger on Iso PVI was ineffective, even after a redo procedure. Among the 6 patients with PV triggers on Ado, PVI and redo PVI failed in one. Of the 6 patients with non-PV triggers on Ado, one was not ablated and one failed PVI and a 
redo. The remaining 4 are without recurrence after the last procedure. Therefore, while Iso was $100 \%$ accurate in predicting a favorable response to PVI, the accuracy of Ado challenge was only 55\%. There was no correlation between non-PV triggers induced by Ado and arrhythmia recurrence after the last procedure $(\mathrm{p}=0.90)$.

\section{Discussion}

The main finding of this study is that, while Iso mostly induces PV triggers, Ado is more likely to induce non-PV triggers of AF, dominantly from the RA. Half of Adoinducible patients had AF from non-PV sites, compared with only $7 \%$ with Iso. Moreover, in two cases the two drugs showed divergent effects in the same patient: Ado inducing non-PV, while Iso PV triggers.

While trigger sites disclosed by Iso challenge showed excellent correlation with the long- term response to PVI (no recurrence in case of PV triggers and recurrence in the single case of non-PV trigger), there was no such correlation seen with Ado, PVI being equally effective in those with PV or non-PV triggers induced by this drug.

\section{Comparison with previous studies}

The use of Iso in the electrophysiology lab to study triggers of AF is well established $(5,6,9)$. It has been shown to effectively identify arrhythmogenic PVs that can selectively be ablated, achieving similar success to empirical 4-PV isolation (11). On the other hand, Ado has mostly been used anecdotally for AF induction. A number of case reports have been published of triggers identified with Ado or ATP, most of which originated outside the PVs (12-17). More investigators have used ATP to test for non-PV triggers after PVI $(18,19,20)$. Strikingly most of these studies originate from Asia, utilize ATP, and report a high rate of non-PV triggers (21). 
Tao et al. (22) used adenosine-triphosphate (ATP) $20 \mathrm{mg}$ for induction in patients with paroxysmal or persistent AF. They found that ATP induced AF in 30\% of the cases and trigger sites were from the PVs in more than $80 \%$ of these. The higher rate of PV-triggering with ATP compared with our results with Ado may be explained by differences in the mode of action and relative doses of the two drugs. The molecular weight of ATP is approximately twice that of Ado; therefore, the number of adenosine molecules in $20 \mathrm{mg}$ ATP is much less than in $18 \mathrm{mg}$ of Ado, the lower dose in our study. On the other hand, ATP exerts a much more pronounced negative chrono- and dromotropic response due to its action on P2 receptors located in the left ventricle inducing a cardiocardiac vagal reflex (23). The higher relative dose and exclusive action on adenosine (P1) receptors may explain the higher rate of RA triggers we saw with Ado.

Nevertheless, another report from Japan using ATP showed a higher rate of RA triggers. Hasebe et al. (23) also used $20 \mathrm{mg}$ of ATP after other methods of AF induction, including Iso, failed. More patients had RA triggers than PV triggers with ATP injection (6 vs. 4) and frequency analysis suggested the driver to reside in the RA during AF in those with RA triggers. These patients were younger and also more often had a family history of AF. The authors suggested they may have a distinct form of the arrhythmia, which they named RA fibrillation. However, it is possible that in Hasebe's study (24), younger patients were more difficult to induce with other methods (including Iso) and therefore more likely to receive ATP or Ado. The higher rate of RA fibrillation in their case might be merely a manifestation of the preferential effect of ATP/Ado on the RA. (24)

The high percentage of RA triggers seen with Ado/ATP may be related to the drug's route of administration, short half-life and mode of action. Ado/ATP is administered 
into a central vein or directly the RA, and therefore the concentration of the drug is higher in the RA than the LA, after having travelled through the lungs, where - due to its very short half-life - a great fraction may be eliminated. Moreover, the sensitivity of the RA to Ado is known to be higher than the LA $(25,26)$, explained by an at least two-fold higher expression of the Ado receptor in the RA (27). Receptor density in the RA has also been correlated to Ado-induced AF in humans (27). Although direct injection of adenosine into the LA had been proposed, this was not done in this study, due to concerns of air embolism (28).

\section{Relation between induced and spontaneous triggers}

Few studies have compared induced and spontaneous AF. Lazar et al. analyzed atrial activation frequency distribution and found no difference between patients with spontaneous AF (3 patients) and Iso-induced AF (13 patients) (29), while Calvo et al. showed the same for pacing induced AF (30). In the abovementioned study, Tao et al. (22) found that the spontaneous AF initiation site was in the PVs in $96 \%$ of cases, while after ATP injection in 85\%. Only in one of the 4 patients with a non-PV trigger manifested by ATP was there a correlation with the spontaneous site. In other words, they show - similarly to our results - more non-PV triggers with ATP, compared with spontaneous AF, but the majority of those ATP-induced non-PV triggers were not clinical. We have seen 4 patients in whom Ado reproduced spontaneous AF, but none originated outside the PVs. Therefore, it seems that Ado/ATP may reproduce PVtriggers; however, the frequent non-PV triggers seen with these drugs cannot be correlated with spontaneous AF.

The question arises whether non-PV triggers induced by Ado are clinically relevant as initiators of spontaneous AF. Triggers of spontaneous AF in our and the above- 
mentioned reports $(4,5,10)$ have been located to the PVs in more than $90 \%$ of the cases. The high percentage of non-PV AF-initiating sites seen with Ado is in sharp contrast with this observation.

\section{Limitations}

The study has several limitations. First, the localization of PV triggers was done with approximation because of the limited number of catheters in the LA and RA. However, differentiating LA from RA and PV from non-PV triggers is straightforward with this catheter setup. After the first drug induced AF, we frequently (33\% after Iso and 22\% after Ado) encountered immediate recurrence of AF after cardioversion preventing us to administer the second drug. Therefore, the number of patients who received both Iso and Ado is relatively low.

\section{Clinical implications}

The literature on non-PV triggers of AF relies heavily on the use of Ado/ATP for induction, although the correlation with spontaneous AF has never been established. We show in a comparison with Iso before PVI that Ado much more frequently induces non-PV triggers, especially from the RA. The clinical significance of these foci, however, remains questionable since Ado induced non-PV triggers in some patients who had PV-triggers with Iso, and the concordance of non-PV triggers with spontaneous AF could not be documented. Moreover, the induction of non-PV triggers by Ado before PVI did not influence the success of the procedure. Based on the above, Ado cannot be recommended for the identification of trigger sites to guide catheter ablation of paroxysmal AF.

This article is protected by copyright. All rights reserved. 
References

1. Haïssaguerre M, Jaïs P, Shah DC, Takahashi A, Hocini M, Quiniou G, Garrigue S, Le Mouroux A, Le Métayer P, Clémenty J.Spontaneous initiation of atrial fibrillation by ectopic beats originating in the pulmonary veins. $\mathrm{N}$ Engl $\mathrm{J}$ Med. 1998 Sep 3;339(10):659-66.

2. Ammar S, Reents T, Fichtner S, Hessling G, Deisenhofer I. Selective Versus Total Pulmonary Vein Isolation In Atrial Fibrillation Ablation. J Atr Fibrillation. 2014 Jun 30;7(1):999.

3. Lin D, Santangeli P, Zado ES Bala R, Hutchinson MD, Riley MP, Frankel DS, Garcia F,Dixit S, Callans DJ, Marchlinski FE. Electrophysiologic findings and long term outcomes in patients undergoing third or more catheter ablation procedures for atrial fibrillation. J Cardiovasc Electrophysiol 2015;26:371-377.

4. Santangeli P, Zado ES, Hutchinson MD, Riley MP, Lin D, Frankel DS, Supple GE, Garcia FC, Dixit S, Callans DJ, Marchlinski FE. Prevalence and distribution of focal triggers in persistent and long-standing persistent atrial fibrillation. Heart Rhythm. 2016 Feb; 13(2):374-82.

5. Oral H, Crawford T, Frederick M, Gadeela N, Wimmer A, Dey S, Sarrazin JF, Kuhne M, Chalfoun N, Wells D, Good E, Jongnarangsin K, Chugh A, Bogun F, Pelosi F Jr, Morady F. Inducibility of paroxysmal atrial fibrillation by isoproterenol and its relation to the mode of onset of atrial fibrillation. $\mathrm{J}$ Cardiovasc Electrophysiol. 2008 May;19(5):466-70.

6. Crawford T, Chugh A, Good E, Yoshida K, Jongnarangsin K, Ebinger M, Pelosi F Jr, Bogun F, Morady F, Oral H.Clinical value of noninducibility by high-dose isoproterenol versus rapid atrial pacing after catheter ablation of paroxysmal atrial fibrillation. J Cardiovasc Electrophysiol. 2010 Jan;21(1):13-20.

This article is protected by copyright. All rights reserved. 
7. Pap R, Tutuianu C, Szilagyi J, Bencsik G, Klausz G, Saghy L, Divergent effect of isoproterenol and adenosine on triggers of paroxysmal atrial fibrillation. Heart Rhythm, Vol.11, No 5, May Supplement 2014, PO04-166

8. Tutuianu C, Traykov V, Bencsik G, Klausz G, Sághy L, Pap R Association between dissociated firing in isolated pulmonary veins and the initiation and maintenance of atrial fibrillation. J IntervCard Electrophysiol. 2016 Jan;45(1):2935.

9. Traykov VB, Pap R, Gingl Z, Chadaide S, Haqqani HM, Klausz G, Gallardo R, Forster T, Callans DJ, Sághy L.Role of triggering pulmonary veins in the maintenance of sustained paroxysmal atrial fibrillation. Pacing Clin Electrophysiol. 2013 Jul;36(7):845-54.

10. Santangeli P, Marchlinski FE Techniques for the provocation, localization, and ablation of non-pulmonary vein triggers for atrial fibrillation Heart Rhythm. 2017 Mar 2. pii: S1547-5271(17)30289-8.

11. Zhang B, Zhen Y, Tao A, Zhang G. Efficacy of selective arrhythmogenic pulmonary veins isolation versus empirical all pulmonary veins isolation for atrial fibrillation: a meta-analysis of randomized and observational studies. J Interv Card Electrophysiol. 2014 Apr;39(3):233-40.

12. Jiang CY, Jiang RH, Matsuo S, Fu GS. ATP revealed extra pulmonary vein source of atrial fibrillation after circumferential pulmonary vein isolation. Pacing Clin Electrophysiol. 2010 Feb;33(2):248-51.

13. Miyazaki S, Takahashi Y, Fujii A, Takahashi A. Adenosine triphosphate exposes multiple extra pulmonary vein foci of atrial fibrillation. Int J Cardiol. 2011 Apr 14;148(2):249-50.

This article is protected by copyright. All rights reserved. 
14. Hioki M, Matsuo S, Yamane T, Tokutake K, Ito K, Narui R, Tanigawa S, Yamashita S, Tokuda M, Inada K, Date T, Yoshimura M. Adenosine-induced atrial tachycardia and multiple foci initiating atrial fibrillation eliminated by catheter ablation using a non-contact mapping system. Heart Vessels. 2012 Mar;27(2):221-6.

15. Knecht S, Castro-Rodriguez J, Janssen C, Verbeet T. Dual utility of adenosine during focal AF. J Cardiovasc Electrophysiol. 2012 Nov;23(11):1264-5.

16. Ip JE, Cheung JW, Chung JH, Liu CF, Thomas G, Markowitz SM, Lerman BB. Adenosine-induced atrial fibrillation: insights into mechanism. Circ Arrhythm Electrophysiol. 2013 Jun;6(3):e34-7.

17. Esato M, Nishina N, Kida Y, Chun Y. A case of paroxysmal atrial fibrillation with a non-pulmonary vein trigger identified by intravenous adenosine triphosphate infusion. J Arrhythm. 2015 Oct;31(5):318-22.

18. Zhang J, Tang C, Zhang Y, Su X. Origin and ablation of the adenosine triphosphate induced atrial fibrillation after circumferential pulmonary vein isolation: effects on procedural success rate. J Cardiovasc Electrophysiol. 2014 Apr;25(4):364-70.

19. Miyazaki S, Kobori A, Hocini M, Shah AJ, Komatsu Y, Taniguchi H, Kusa S, Uchiyama T, Nakamura H, Hachiya H, Isobe M, Hirao K, Haïssaguerre M, Takahashi A, Iesaka Y. Clinical utility of adenosine-infusion test at a repeat atrial fibrillation ablation procedure. Heart Rhythm. 2013 May;10(5):629-35.

20. Hayashi K, An Y, Nagashima M, Hiroshima K, Ohe M, Makihara Y, Yamashita K, Yamazato S, Fukunaga M, Sonoda K, Ando K, Goya M. Importance of nonpulmonary vein foci in catheter ablation for paroxysmal atrial fibrillation. Heart Rhythm. 2015 Sep;12(9):1918-24.

This article is protected by copyright. All rights reserved. 
21. Belhassen B, Michowitz Y. Unmasking right atrial fibrillation: A new indication of adenosine triphosphate test? Heart Rhythm. 2016 Feb;13(2):364-5.

22. Tao S, Yamauchi Y, Maeda S, Okada H, Yamaguchi T, Hara N, Konishi Y, Umemoto T, Miyamoto T, Obayashi T, Hirao K, Isobe M. Adenosine triphosphate induced atrial fibrillation:the clinical significance and relevance to spontaneous atrial fibrillation J Interv Card Electrophysiol. 2014 Mar; 39(2): 103 -9.

23. Pelleg A, Belhassen B. The mechanism of the negative chronotropic and dromotropic actions of adenosine 5'-triphosphate in the heart: an update. J Cardiovasc Pharmacol. 2010 Jul;56(1):106-9.

24. Hasebe H, Yoshida K, Iida M, Hatano N, Muramatsu T, Aonuma K Right-to-left frequency gradient during atrial fibrillation initiated by right atrial ectopies and its augmentation by adenosine triphosphate: Implications of right atrial fibrillation.Heart Rhythm. 2016 Feb;13(2):354-63.

25. Nakai T, Watanabe I, Kunimoto S, Kojima T, Kondo K, Saito S, Ozawa Y, Kanmatsuse K. Electrophysiological effect of adenosinetriphosphate and adenosine on atrial and ventricular action potential duration in humans. Jpn Circ J. 2000 Jun;64(6):430-5.

26. Botteron GW, Smith JM. Spatial and temporal inhomogeneity of adenosine's effect on atrial refractoriness in humans: using atrial fibrillation to probe atrial refractoriness. J Cardiovasc Electrophysiol. 1994 Jun;5(6):477-84.

27. Li N, Csepe TA, Hansen BJ, Sul LV, Kalyanasundaram A, Zakharkin SO, Zhao J, Guha A, Van Wagoner DR, Kilic A, Mohler PJ, Janssen PM, Biesiadecki BJ, Hummel JD, Weiss R, Fedorov VV. Adenosine-Induced Atrial Fibrillation: Localized Reentrant Drivers in Lateral Right Atria due to Heterogeneous 
Expression of Adenosine A1 Receptors and GIRK4 Subunits in the Human Heart.

Circulation. 2016 Aug 9;134(6):486-98.

28. Cha MJ, Choi EK, Oh S. Comparison between local and systemic injection of adenosine for detecting dormant conduction after PV isolation. Pacing Clin Electrophysiol. 2017 Jul;40(7):762-769.

29. Lazar S, Dixit S, Marchlinski FE, Callans DJ, Gerstenfeld EP. Presence of left-toright atrial frequency gradient in paroxysmal but not persistent atrial fibrillation in humans. Circulation. 2004 Nov 16; 110(20):3181-6.

30. Calvo D, Atienza F, Jalife J, Martínez-Alzamora N, Bravo L, Almendral J, González-Torrecilla E, Arenal Á, Bermejo J, Fernández-Avilés F, Berenfeld O. High-rate pacing-induced atrial fibrillation effectively reveals properties of spontaneously occurring paroxysmal atrial fibrillation in humans. Europace. 2012 Nov;14(11):1560-6.

Figure 1. Left anterior oblique (LAO) and right anterior oblique (RAO) view showing the two decapolar catheters placed in the coronary sinus (CS) and right atrium (RA), circular mapping catheter (CMC) in the left pulmonary veins (PVs) and mapping catheter (ABL) in right PVs.

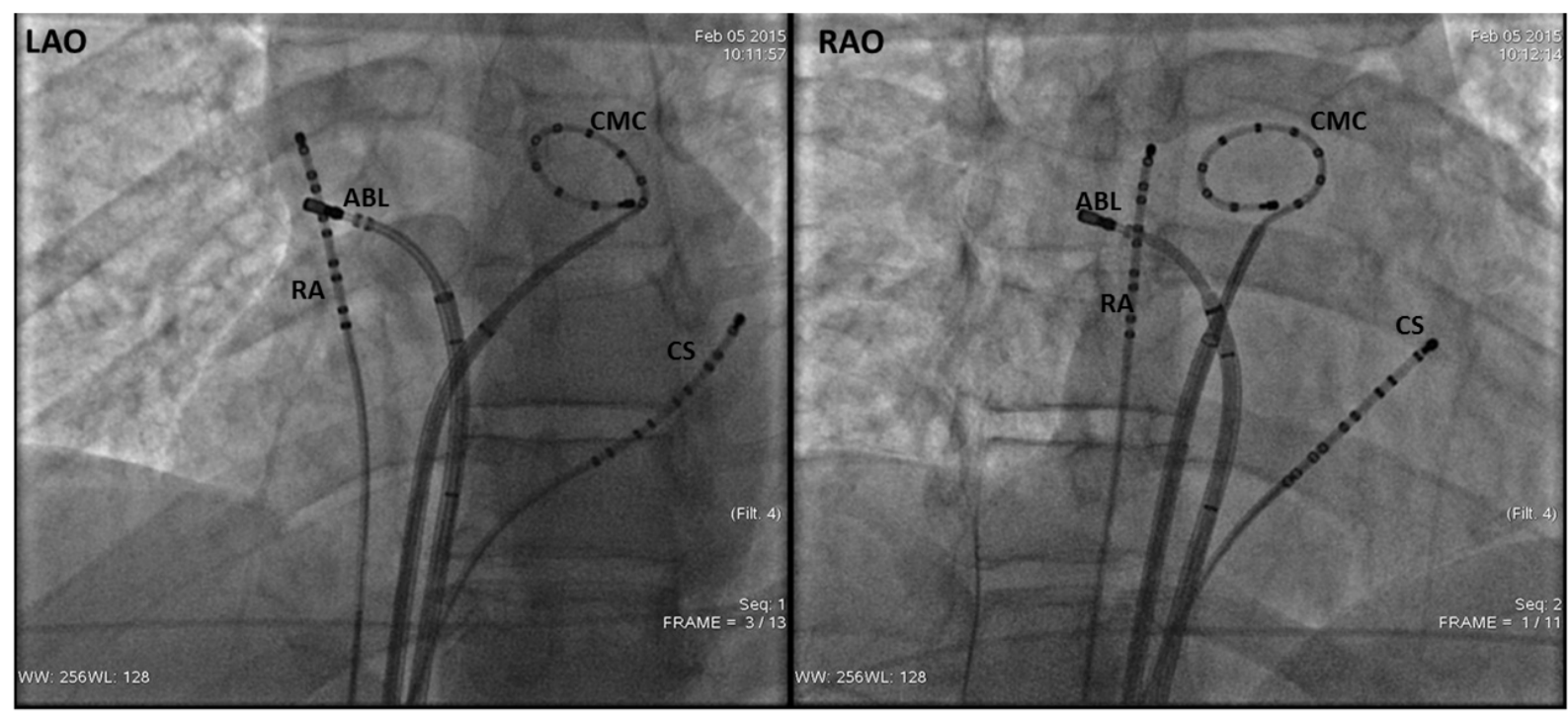

This article is protected by copyright. All rights reserved. 
Figure 2. Surface electrocardiogram lead I, II, V6 along with intracardiac recordings from right atrium (RA), coronary sinus (CS) and circular mapping catheter (CMC) placed in the pulmonary vein (PV) antrum, during initiation of atrial fibrillation. Panel (A) during adenosine infusion AF was induced from RA. Spontaneously (Panel B) and during isoproterenol infusion (Panel C) AF was induced from the PVs. Paper speed=75mm/s. During redo procedure only the previous triggering pulmonary vein was reconnected (Panel D). Paper speed $=100 \mathrm{~mm} / \mathrm{s}$.
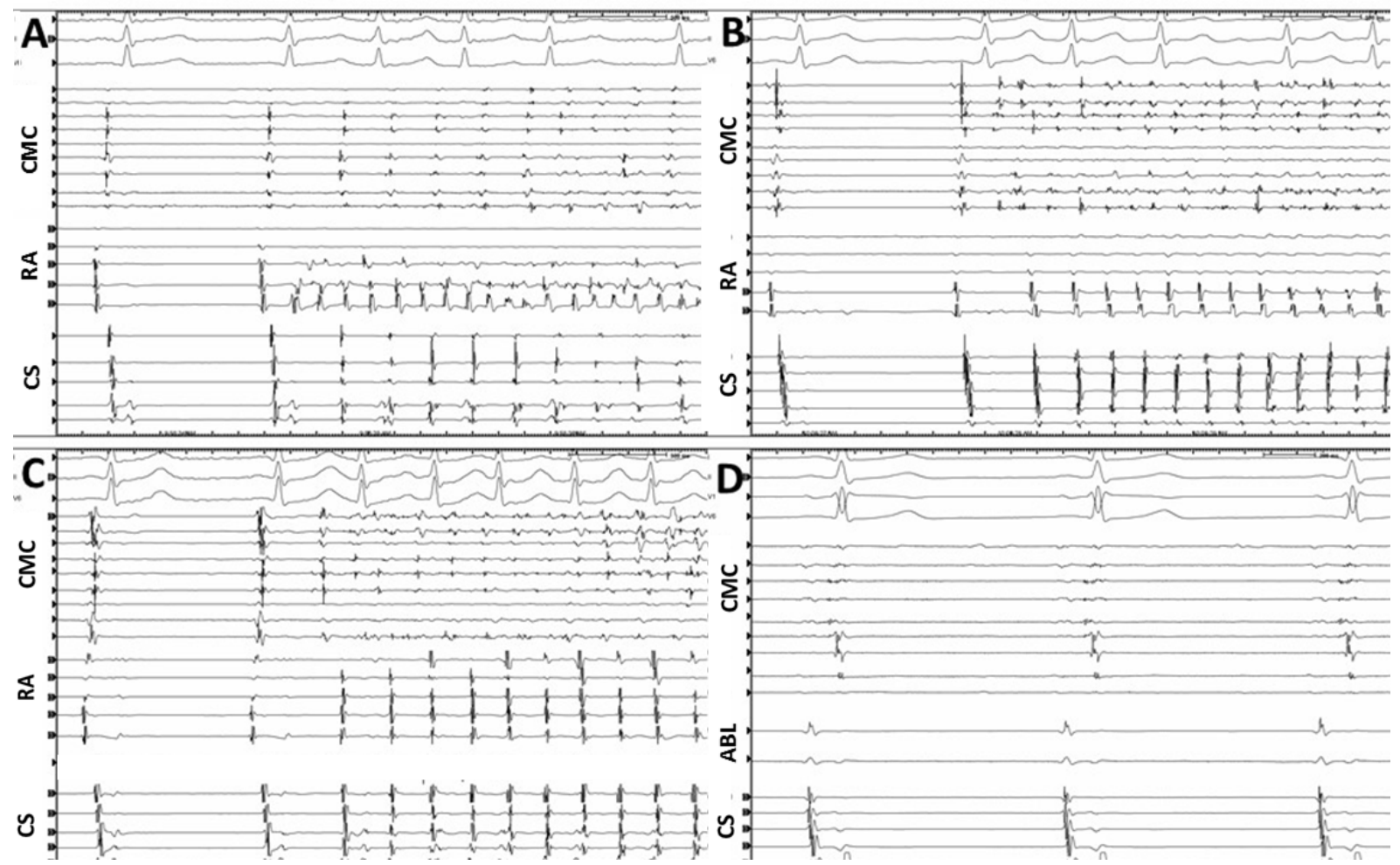

Figure 3. Trigger sites of isoproterenol induced AF (Panel A) and adenosine induced AF (Panel B).
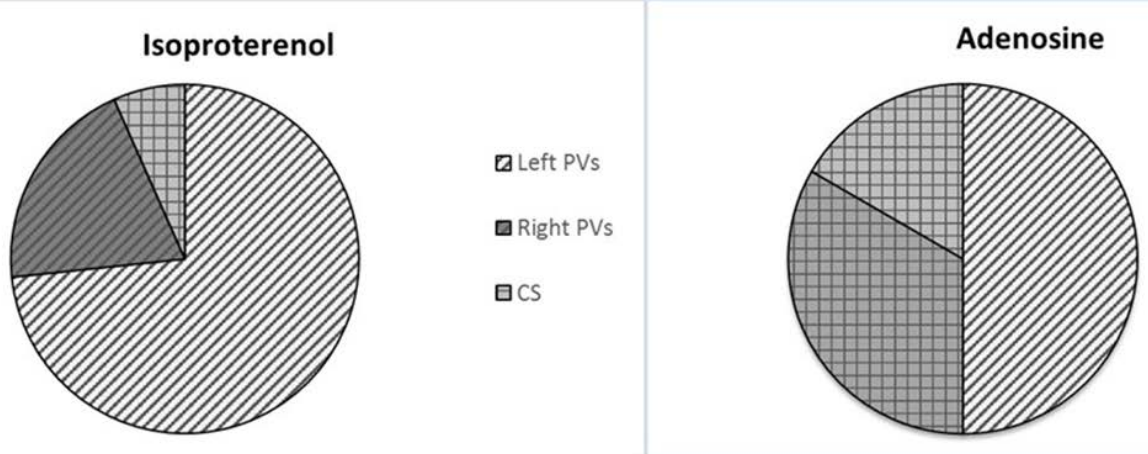

$\square$ Left PVs
$\square R A$
$\square C S$

This article is protected by copyright. All rights reserved. 
Figure 4. Study flowchart. IRAF: immediate recurrence of atrial fibrillation (AF) after cardioversion, Ado: adenosine, Iso: isoproterenol, LPV: left pulmonary veins (PV), RPV: right $\mathrm{PV}, \mathrm{RA}$ : right atrium, CS: coronary sinus.

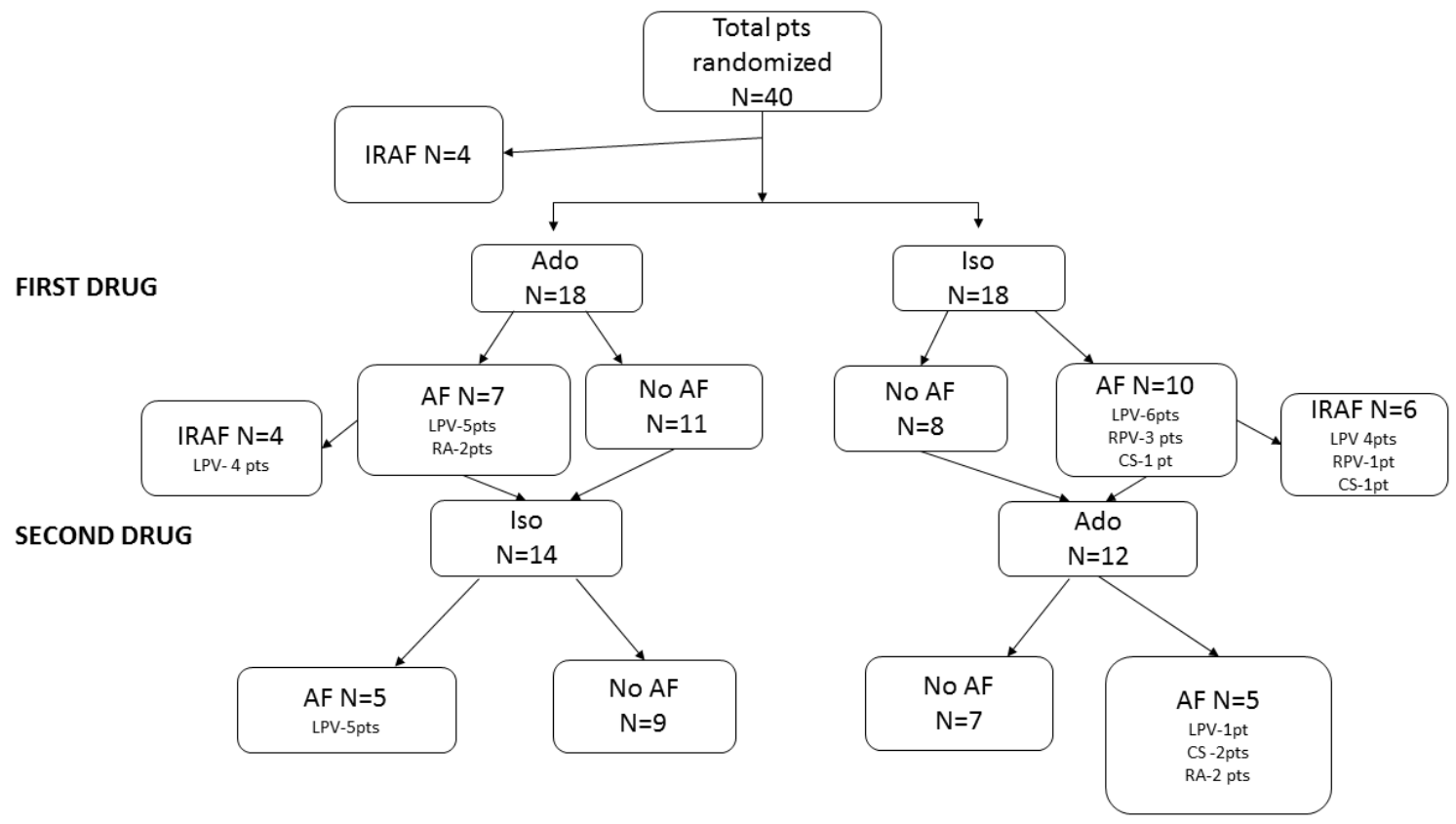

Table 1. Characteristics of patients who received both drugs. Abbreviations are as on Figure 4.

\begin{tabular}{|c|c|c|c|c|c|c|}
\hline \multirow{2}{*}{ Patient \# } & \multirow{2}{*}{ First drug } & \multicolumn{2}{|c|}{ Isoproterenol } & \multicolumn{2}{c|}{ Adenosine } & \multirow{2}{*}{$\begin{array}{c}\text { Spontaneous } \\
\text { triggers }\end{array}$} \\
\cline { 3 - 7 } & & $\begin{array}{c}\text { Max. dose } \\
(\mu \mathrm{g} / \mathrm{min})\end{array}$ & $\begin{array}{c}\text { Induced } \\
\text { triggers }\end{array}$ & $\begin{array}{c}\text { Max. dose } \\
(\mathrm{mg})\end{array}$ & $\begin{array}{c}\text { Induced } \\
\text { triggers }\end{array}$ & \\
\hline 1 & Ado & 10 & LPV & 18 & RA & - \\
\hline 2 & Ado & 20 & LPV & 18 & LPV & LPV \\
\hline 3 & Iso & 20 & LPV & 18 & LPV & - \\
\hline 4 & Iso & 20 & - & 18 & RA & - \\
\hline 5 & Ado & 20 & - & 18 & RA & - \\
\hline 6 & Ado & 20 & LPV & 36 & - & - \\
\hline 7 & Ado & 20 & LPV & 36 & - & \\
\hline
\end{tabular}

This article is protected by copyright. All rights reserved. 


\begin{tabular}{|c|c|c|c|c|c|c|}
\hline 8 & Iso & 5 & LPV & 36 & CS & - \\
\hline 9 & Ado & 20 & LPV & 36 & - & - \\
\hline 10 & Iso & 15 & RPV & 36 & - & RPV \\
\hline 11 & Iso & 3 & LPV & 36 & - & LPV \\
\hline 12 & Iso & 20 & - & 36 & - & - \\
\hline 13 & Ado & 20 & - & 36 & - & - \\
\hline 14 & Iso & 20 & - & 36 & - & - \\
\hline 15 & Ado & 20 & - & 36 & - & LPV \\
\hline 16 & Ado & 20 & - & 36 & - & - \\
\hline 17 & Ado & 20 & - & 36 & - & - \\
\hline 18 & Ado & 20 & - & 36 & - & - \\
\hline 19 & Iso & 20 & - & 36 & - & - \\
\hline 20 & Iso & 20 & - & 36 & - & - \\
\hline 21 & Iso & 20 & - & 36 & - & - \\
\hline 22 & Ado & 20 & - & 36 & - & - \\
\hline 23 & Iso & 20 & - & 36 & CS & - \\
\hline 24 & Ado & 20 & - & 36 & - & - \\
\hline 25 & Iso & 20 & - & 36 & RA & - \\
\hline 26 & Ado & 20 & - & 36 & - & - \\
\hline
\end{tabular}

This article is protected by copyright. All rights reserved. 